\title{
Research on the Power System Frequency Measurement Based on Support Vector Machine
}

\author{
Liu Jiashuo \\ Department of Electrical Engineering, North China Electric Power University, Baoding, China
}

Keywords: Support vector machine, Power system, Frequency measurement, FFT

\begin{abstract}
Power system frequency generates an important impact on operation, control and protection of the power system, and it is the important index of weighing power quality, so it is very necessary to measure it accurately. First, this paper uses the linear regression function to fit the signal including harmonic needed to be measured and the iterative reweighted least squares procedure is educed for signal spectrum analysis. Then, a new method to measure power system frequency is presented. In case of the few sample number, this method, using single frequency to sound out whether it can fit the original signal curve well or not, can measure power system frequency with accuracy by comparing different amplitude. Through discussing three examples and com paring with FFT, it can be proved that the new method has the characteristics of simple computation and high precision.
\end{abstract}

\section{Introduction}

In the power system measurement device, the measurement of input signal is mostly used in direct exchange sampling, and the instantaneous value of the measured signal is sampled, analyzed and calculated to obtain the measured information [1-2]. At present, the main factor affecting the exchange sampling accuracy is that the base wave frequency of input signal cannot be measured in real time [3]. There are theoretically many ways to measure the frequency of the power system well:

Cycle Method. The original cycle method calculates the frequency by measuring the time width between successive zero crossings of the signal waveform. The improved algorithm has horizontal algorithm, high order correction algorithm and the least polynomial curve fitting algorithm.

Analytical Method. The signal observation model is mathematically transformed and the $f$ or $\Delta f$ to be measured is expressed as an explicit function of the sample value.

Error minimization Algorithm. Using a noisy signal observation model, the algorithm involves a certain norm that minimizes the error. Since the algorithms are described in detail in the areas of mathematical analysis and signal processing, the crux of the problem lies in solving the measurement into the corresponding standard format, and reduce the amount of computation.

FFT Algorithm and Improved Algorithm. FFT is a kind of typical digital filtering technology. When the sampling frequency and data window are chosen properly, the filtering algorithm can get the model parameters correctly. Taking into account the real measurement deviates from the ideal conditions, the use of front-end window FFT results to estimate the system's fundamental frequency.

Above these frequency measurement method, some are of low accuracy, affected by the harmonic and noise and aperiodic component, the real-time performance of some is not good, others contain complex mathematical deduction, and theirs implementation is difficult [4-5]. The methods can truly applied in the engineering practice is less. This paper applies support vector machine (SVM) method to accurately detect the frequency of power system, which has the characteristics of high detection accuracy, easy implementation, and strong harmonic ability.

\section{Support Vector Machine}

Support vector machine is a sophisticated and highly principled learning method for designing a sub-linear element only contained in the hidden layer of back forward network. SVM is based on 
the risk minimization principle of VC dimension theory. It can overcome many shortcomings of traditional methods and neural network model. The prior knowledge of specific issues are not required. In the case of limited training samples, it can effectively control the machine learning ability. The basic idea of SVM for classification is that samples in the input space will be mapped to a feature space through a non-linear relationship, then the optimal hyperplane will be constructed in the proposed space, which can classify the samples easily.

Model Establishment. For support vector machine function fitting, first consider the use of linear regression function $f(x)=w x+b$. The fitting data is $\left\{x_{i}, y_{i}\right\}, i=1,2, \cdots, n, x_{i} \in R^{d}$, $y_{i} \in R$.

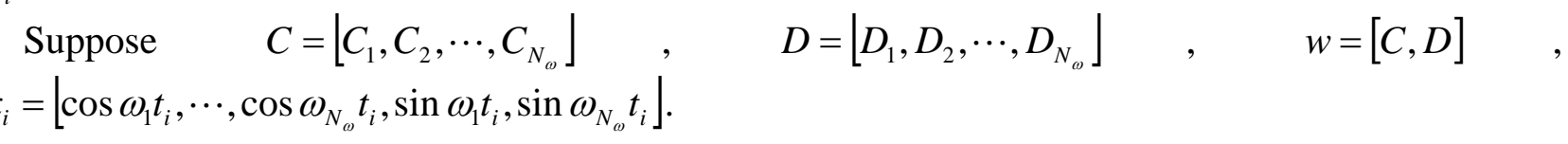

This problem translates into solving the frequency decomposition model of the discrete time series $\left\{t_{i}, y_{i}\right\}, i=1,2, \cdots, N$ :

$$
y_{i}=\sum_{m=1}^{N_{\omega}} A_{m} \cos \left(\omega_{m} t_{i}-\varphi_{m}\right)+e_{i}=\sum_{m=1}^{N_{\omega}}\left(C_{m} \omega_{m} t_{i}+D_{m} \sin \omega_{m} t_{i}\right)+e_{i}
$$

Where $A_{m}$ and $\varphi_{m}$ are the magnitude and phase angle of the components of the angular frequency $\omega_{m}$, respectively, $e_{i}$ is the model error at time $t_{i}, e_{i}=y_{i}-w x_{i}^{T}, C_{m}=A_{m} \cos \varphi_{m}$, $D_{m}=A_{m} \sin \varphi_{m}$. It can be seen, when the signal contains a component of angular frequency $\omega_{m}$, $A_{m} \neq 0$. The objective function of this model can be expressed as:

$$
\min \frac{1}{2} \sum_{m=1}^{N_{\omega}}\left(C_{m}^{2}+D_{m}^{2}\right)+\frac{c}{2} \sum_{i=1}^{N}\left(\xi_{i}^{(k)}+\xi_{i}^{*(k)}\right)
$$

Constraints are:

$$
\left\{\begin{array}{l}
y_{i}-\sum_{m=1}^{N_{\omega}}\left(C_{m} \cos \omega_{m} t_{i}+D_{m} \sin \omega_{m} t_{i}\right) \leq \varepsilon+\xi_{i} \\
\sum_{m=1}^{N_{\omega}}\left(C_{m} \cos \omega_{m} t_{i}+D_{m} \sin \omega_{m} t_{i}\right)-y_{i} \leq \varepsilon+\xi_{i}^{*} \\
\xi_{i}, \xi_{i}^{*} \geq 0
\end{array}\right.
$$

The first term in Formula 2 makes the function more flat and improves the generalization ability. The second term is the loss function, where $c$ is the fault-tolerant penalty function and $\xi_{i}$ and $\xi_{i}^{*}$ are the slack variables, which solve the problem that some point errors are greater than $\varepsilon . \xi^{(k)}$ represents $k$ times loss function. The commonly used loss functions in SVM regression are the linear $\varepsilon$ insensitive loss function, the quadratic $\varepsilon$ insensitive loss function and the Huber loss function. In actual use, the appropriate loss function is selected according to the characteristics of the actual model. In this paper, we choose the quadratic $\varepsilon$ insensitive loss function:

$$
\begin{aligned}
& \xi^{(2)}=\left\{\begin{array}{l}
0, y-f(x) \leq \varepsilon \\
|y-f(x)|^{2}-\varepsilon, y-f(x) \geq \varepsilon
\end{array}\right. \\
& \xi^{*(2)}=\left\{\begin{array}{l}
0, y-f(x) \geq-\varepsilon \\
|y-f(x)|^{2}-\varepsilon, y-f(x) \leq-\varepsilon
\end{array}\right.
\end{aligned}
$$

Parameters Solution. If $\xi_{i}^{(*)}<0$, then set $\xi_{i}^{(*)}=0$. The first two constraints in Formula (3) remain. This change will reduce the value of the objective function. The optimal solution obtained by removing the positive constraint $\xi_{i}^{(*)}$ is consistent with the solutions of Formula (2) and (3). Therefore, Lagrange function is introduced for the optimization problem of Formula (2) and (3), 
which can be expressed as:

$$
L=\frac{1}{2}\|w\|^{2}+\frac{c}{2} \sum_{i=1}^{N}\left(\xi_{i}^{(2)}+\xi_{i}^{*(2)}\right)+\sum_{i=1}^{N} \alpha_{i}\left(y_{i}-w x_{i}^{T}-\varepsilon-\xi_{i}\right)+\sum_{i=1}^{N} \alpha_{i}^{*}\left(w x_{i}^{T}-y_{i}-\varepsilon-\xi_{i}^{*}\right)
$$

Where $\alpha_{i}$ and $\alpha_{i}^{*}$ are Lagrange constant. According to $\left(\partial L / \partial \xi_{i}^{(*)}\right)=0$, there has $c\left(\xi_{i}^{(*)}+\varepsilon\right)-\alpha_{i}^{(*)}=0$. Then set $\lambda_{i}=2 \alpha_{i} /\left(e_{i}-\varepsilon\right)$, then there has

$$
\begin{aligned}
& \lambda_{i}=\left\{\begin{array}{l}
0, e_{i} \leq \varepsilon \\
2 c e_{i} /\left(e_{i}-\varepsilon\right), \text { else }
\end{array}\right. \\
& \lambda_{i}^{*}=\left\{\begin{array}{l}
0, e_{i} \geq-\varepsilon \\
-2 c e_{i} /-\left(e_{i}+\varepsilon\right), \text { else }
\end{array}\right. \\
& L=\frac{1}{2}\|w\|^{2}+\frac{1}{2} \sum_{i=1}^{N}\left(\lambda_{i}\left(e_{i}-\varepsilon\right)^{2}+\lambda_{i}^{*}\left(-e_{i}-\varepsilon\right)^{2}\right)+\frac{c}{2} \sum_{i=1}^{N}\left(\xi_{i}^{2}+\xi_{i}^{* 2}\right)-\sum_{i=1}^{N}\left(\alpha_{i} \xi_{i}+\alpha_{i}^{*} \xi_{i}^{*}\right)
\end{aligned}
$$

Since each iteration process, $\lambda_{i}^{(*)}$ and $\alpha_{i}^{(*)}$ are constant, then there has

$$
\frac{\partial L}{\partial w}=w-\sum_{i=1}^{N} x_{i}^{T}\left(\lambda_{i}+\lambda_{i}^{*}\right) e_{i}+\sum_{i=1}^{N} x_{i}^{T}\left(\lambda_{i}-\lambda_{i}^{*}\right) \varepsilon=w+X D_{\left(\lambda_{i}+\lambda_{i}^{*}\right)} w X^{T}-x D_{\left(\lambda_{i}+\lambda_{i}^{*}\right)} y+X D_{\left(\lambda_{i}-\lambda_{i}^{*}\right)} \varepsilon
$$

Here,

$$
\begin{aligned}
& X=\left[x_{1}^{T}, x_{2}^{T}, \cdots, x_{i}^{T}\right] \\
& D_{\left(\lambda_{i}+\lambda_{i}^{*}\right)}=\left[\begin{array}{cccc}
\lambda_{1}+\lambda_{1}^{*} & 0 & \cdots & 0 \\
0 & \lambda_{2}+\lambda_{2}^{*} & 0 & \vdots \\
\vdots & 0 & \ddots & 0 \\
0 & \cdots & 0 & \lambda_{i}+\lambda_{i}^{*}
\end{array}\right]
\end{aligned}
$$

Where $D_{\left(\lambda_{i}-\lambda_{i}^{*}\right)}$ is similar with Formula (12), from $\partial L / \partial w=0$, the following can be obtained:

$$
w=\left[I+X D X^{T}\right]^{-1} X\left[D_{\left(\lambda_{i}+\lambda_{i}^{*}\right)} y-D_{\left(\lambda_{i}-\lambda_{i}^{*}\right)} \varepsilon\right]
$$

Algorithm Steps. From the above derivation can be summed up to solve the IRWLS method is:

(1) Set the initial value of the adopted frequency, sampling points and random sequence $w$, and set the iteration number $r=0$.

(2) Calculate $e_{i}, \alpha_{i}$ and $\alpha_{i}^{*}$.

(3) Solve $\lambda_{i}$ and $\lambda_{i}^{*}$.

(4) Solve $w_{r+1}$ and set $r=r+1$.

(5) Judge $\left\|w_{r+1}-w_{r}\right\|<0.1$, stop the operation for real, or jump to step (2).

\section{Case study}

It can be seen that $\omega_{m}$ and $N_{\omega}$ in Formula (1) can be set arbitrarily. Since the range of the frequency in the power system is definite, $N_{\omega}=1$ can be tentatively set in this range, and different values $\omega_{m}$ find the power system frequency. The following three examples show that the algorithm can accurately detect the power system frequency in the case of fewer sampling points, and compared with the FFT.

The Condition of no Harmonics in the Signal. Set the signal as $x=\sin (51 \times 2 \pi t-\pi / 6)$, the sampling frequency is $300 \mathrm{~Hz}$, and the sampling points is 6 . Suppose $N_{\omega}=1$, the frequency $f\left(f=\omega_{m} / 2 \pi\right)$ is set to one of $45 \mathrm{~Hz}$ to $55 \mathrm{~Hz}$, respectively. The simulation results are shown in Table 1. 
Table 1. SVM simulation results

\begin{tabular}{cccccc}
\hline$f / \mathrm{Hz}$ & 46 & 47 & 48 & 49 & 50 \\
Amplitude/V & 0.5408 & 0.5437 & 0.5458 & 0.5471 & 0.5478 \\
\hline$f / \mathrm{Hz}$ & 51 & 52 & 53 & 54 & 55 \\
Amplitude/ $\mathrm{V}$ & 0.5478 & 0.5472 & 0.5460 & 0.5442 & 0.5419 \\
\hline
\end{tabular}

It can be seen that when $\omega_{m}$ takes different values, the amplitudes obtained are different, and the amplitude is the largest when $\omega_{m}$ is $51 \mathrm{~Hz}$, which shows that the curve fits best at this time. The result is a frequency of $51 \mathrm{~Hz}$.

In the same situation, the application of FFT simulation results are shown in Figure 1. It can be seen that the FFT algorithm can not get accurate frequency under this condition. Because of the frequency resolution of $51 \mathrm{~Hz}$, the FFT must have a frequency resolution of $1 \mathrm{~Hz}$, which theoretically requires a sampling time of at least 1 second (50 cycles). Then at a sampling frequency of $300 \mathrm{~Hz}$, the number of samples should be increased to at least 300 .

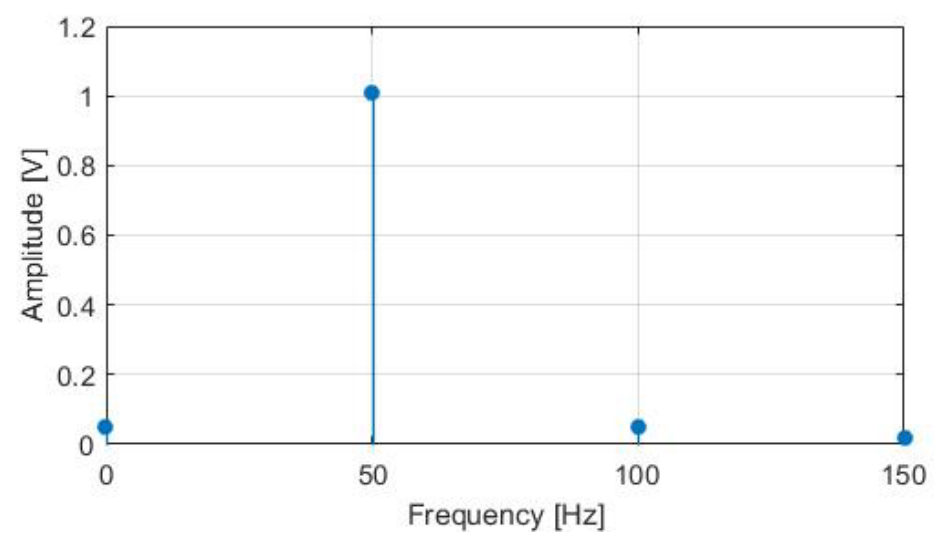

Figure 1. FFT simulation results

The Condition of Containing Harmonics in the Signal. In the actual measurement of power system frequency, the high-pass filter is usually used to attenuate the high harmonics to a negligible level, so that the impact of measurement is mainly the second and third harmonics. The following signal containing the second and third harmonics is to discuss.

Set the signal as $x=\sin (2 \pi f t-\pi / 6)+0.2 \sin (4 \pi f t-\pi / 7)+0.1 \sin (6 \pi f t-0.1 \pi)$, where $f$ is $51 \mathrm{~Hz}$, the sampling frequency is $300 \mathrm{~Hz}$, and the sampling points is 14 . Suppose $N_{\omega}=1$, the frequency $f$ is set to one of $45 \mathrm{~Hz}$ to $55 \mathrm{~Hz}$. The simulation results are shown in Table 2. The simulation results show that the frequency can be detected accurately when the signal contains two or three harmonics. The results of the FFT simulation are shown in Figure 2.

The Condition of Signal Frequency Detection Accuracy to $0.1 \mathrm{~Hz}$. In the above two cases, the accuracy reaches $1 \mathrm{~Hz}$. When the accuracy needs to reach $0.1 \mathrm{~Hz}$, the method is equally effective and requires increasing the number of sampling points. Example 3 will be described below. Set the signal as $x=\sin (2 \pi \times 50.2 \times t-\pi / 6)$, the sampling frequency is $300 \mathrm{~Hz}$, and the sampling points is 66. Suppose $N_{\omega}=1$, the frequency $f$ is set to one of $49.7 \mathrm{~Hz}$ to $50.4 \mathrm{~Hz}$. The simulation results are shown in Table 3.

Table 2. SVM simulation results

\begin{tabular}{cccccc}
\hline$f / \mathrm{Hz}$ & 46 & 47 & 48 & 49 & 50 \\
Amplitude/V & 0.6563 & 0.6891 & 0.6147 & 0.7322 & 0.7412 \\
\hline$f / \mathrm{Hz}$ & 51 & 52 & 53 & 54 & 55 \\
Amplitude/V & 0.7420 & 0.7356 & 0.7229 & 0.7054 & 0.6841 \\
\hline
\end{tabular}




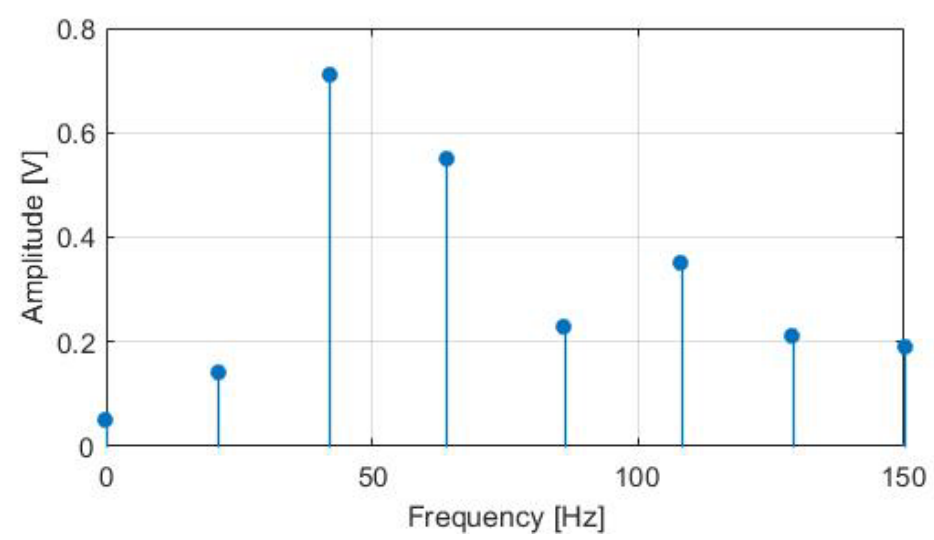

Figure 2. FFT simulation results

Table 3. SVM simulation results

\begin{tabular}{ccccccccc}
\hline$f / \mathrm{Hz}$ & 49.7 & 49.8 & 49.9 & 50.0 & 50.1 & 50.2 & 50.3 & 50.4 \\
\hline Amplitude/V & 0.912 & 0.919 & 0.924 & 0.927 & 0.929 & 0.930 & 0.929 & 0.926 \\
\hline
\end{tabular}

It is obvious that when $f$ is $50.2 \mathrm{~Hz}$, the largest amplitude is obtained. Therefore, the proposed method can accurately detect the signal frequency of $0.1 \mathrm{~Hz}$. The results of the FFT simulation are shown in Figure 3.

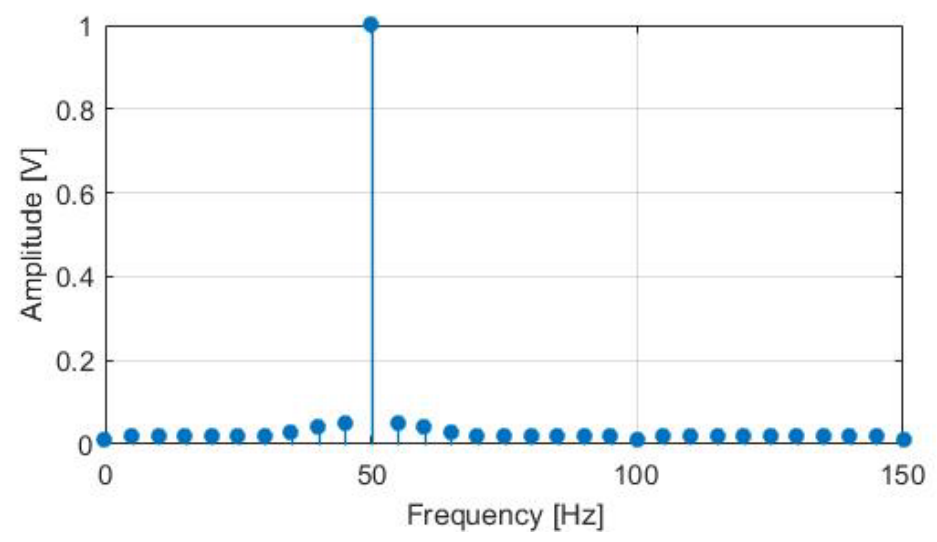

Figure 3. FFT simulation results

In fact, if the FFT method is used to achieve the frequency resolution to reach $0.1 \mathrm{hz}$, the minimum sampling is 10 seconds (500 cycles), which is obviously intolerable in practical engineering application.

\section{Conclusion}

The new method of detecting power system frequency based on SVM algorithm proposed in this paper can eliminate the influence of the second and third harmonics. SVM is the updated and more effective part of statistical learning theory, and the core of it was put forward from 1992 to 1995 and is still in constant developing stage at present. The theory based on the principle of structure risk minimization provides a new perspective in machine learning, and has been widely applied to pattern recognition and function fitting. When the sampling points are few, the power system frequency is accurately detected, which has the characteristics of simple calculation and high accuracy.

\section{References}

[1] Huang Chun, He Yigang, Jiang Yaqun, et al. Analysis and improvement of synchronization 
technique for AC sampling[J]. Proceeding s of the CSEE, 2002, 22(9): 38- 42.

[2] Sounan Jiale, Ge Yaozhong, Wang Anding , et al. New algorithm avoiding voltage cross-zero point for measuring the frequency[J]. Proceedings of the CSU-EPSA, 1996, 8(4): 18-22.

[3] Li Zhenran. Using the recursive least square algorithm and adaptive sampling to realize micro computer electrical quantity transducer [J]. Automation of Electric Power System, 1995, 19(3): 48-52.

[4] Xie Xiaorong, Han Yingduo. A overview on power system frequency measurement [J]. Automation of Electric Power System, 1999, 23(3): 54- 58.

[5] José Luis Rojo-lvarez, Manel M artinez-Ramón , A nibal R FigueirasVidal, et al. A robust support vector algorithm for nonparametric spectral analysis[J]. IEEE Signal Processing Letter, 2003, 10(11):320-323. 